\title{
PERCEPÇÃO DA QUALIDADE ALIMENTAR ENTRE OS USUÁRIOS DE UMA CLÍNICA-ESCOLA NO INTERIOR DA BAHIA, BRASIL
}

\author{
Alciene Pereira da Silva \\ União Metropolitana de Educação e Cultura - UNIME/Itabuna \\ alcieneps@gmail.com \\ Roque Pinto \\ Universidade Estadual de Santa Cruz \\ roquepintosantos@gmail.com
}

\section{Resumo}

Este trabalho pretende descrever e analisar as atividades extensionistas de educação em saúde com foco no aspecto alimentar, realizadas junto à população do entorno de uma clínica escola localizada no sul do Estado da Bahia, Brasil. As atividades foram iniciadas em março e finalizadas em julho de 2015, atendendo um total de 570 pessoas, e consistiram na aplicação de um questionário e na realização de três oficinas desenhadas a partir das respostas dos questionários e direcionadas para conhecer os hábitos alimentares dos participantes e sugerir novas condutas que pudessem vir a auxiliar na promoção à saúde. Os resultados finais apontaram que os indivíduos com menor renda e escolaridade tendem a não considerar as informações dos rótulos dos produtos alimentares e a conhecer menos os riscos de uma má alimentação à sua saúde.

Palavras-chave: Educação em saúde. Padrões alimentares. Rotulagem. Consumo alimentar.

\section{PERCEPTION OF FOOD QUALITY AMONG USERS OF A CLINIC-SCHOOL IN THE INTERIOR OF THE STATE OF BAHIA, BRAZIL}

\begin{abstract}
This work intends to describe and analyze the extensionist activities of health education with focus on the food aspect, carried out with to the population of the surroundings of a school clinic located in the south of the State of Bahia, Brazil. The activities started in March and were completed in July 2015, attending a total of 570 people, and consisted in the application of a survey and the accomplishment of three workshops designed from the answers of the survey and directed to know the eating habits of the participants and suggest new behaviors that could help promote health. The final results pointed out that individuals with lower income and schooling tend not to consider food label information and to know less about the risks of poor diet to their health.
\end{abstract}

Key-words: Health education. Food standards. Labeling. Food consumption.

\section{PERCEPCIÓN DE LA CALIDAD ALIMENTARIA ENTRE LOS USUARIOS DE UNA CLÍNICA-ESCUELA EN EL INTERIOR DE LA PROVINCIA DE BAHIA, BRASIL}

\begin{abstract}
Resumen
Este trabajo pretende describir y analizar las actividades de extensión en educación para la salud con enfoque en el aspecto alimentario, realizado con la población del entorno de una clínica escolar ubicada en el sur de la Provincia de Bahia, Brasil. Las actividades empezaron en marzo y se completaron en julio de 2015, atendiendo a un total de 570 personas, y consistieron en la aplicación de una encuesta y la realización de tres talleres diseñados a partir de las respuestas de la encuesta, con el objetivo de conocer los hábitos alimenticios de los participantes y proponer nuevas conductas que puedan ayudar a promover la salud. Los resultados finales señalaron que las personas con bajos ingresos y baja escolaridad tienden a no considerar la información de la etiqueta de los alimentos y saber menos acerca de los riesgos de una mala alimentación para su salud.

Palabras clave: Educación en salud. Patrones alimentarios. Etiquetado. Consumo alimentario.
\end{abstract}


Percepção da qualidade alimentar entre os usuários de uma clínica-escola no interior da Bahia, Brasil

\section{INTRODUÇÃO}

$\mathrm{O}$ ato de comer para os humanos não se resume à mera administração de nutrientes e calorias para a manutenção do funcionamento corporal. A ingestão de alimentos está relacionada ao contexto material, sociocultural e simbólico. Refere-se, portanto, simultaneamente a uma exigência físico-biológica e a uma experiência subjetiva inscritas em um plano cultural mais amplo em que são anotadas questões de naturezas diversas (CANESQUI e GARCIA, 2005; ROMANELLI, 2006; KAC, SICHIERI e GIGANTE, 2007).

$\mathrm{Na}$ interface entre as ciências da saúde e as ciências sociais diversos autores vêm atuando em investigações que apontam para uma série de fatores e variáveis que contribuem para o estabelecimento de padrões de consumo ou padrões alimentares (NAJAS et al, 1994; OLINTO, 2007; MUNIZ et al, 2015; POSSA et al, 2017) com consequências verificáveis na saúde da população ( BEZERRA et al, 2014; COELHO et al, 2015; LIMA et al, 2015; ANJOS, 2006).

Assim, as substâncias elegíveis como alimentos têm um peso importante no processo saúde e doença e figuram numa relação muito mais complexa do que suporia um modelo mecânico de contenções físicas entre o homem e os agentes patógenos (BOOG, 1999; PINHEIRO et al, 2005; LAPLANTINE, 2010; MENDES, 2012; BRASIL, 2013). É nesta perspectiva complexa e multifacetada que a dimensão alimentar cobra importância, na medida em que não pode ser apartada dos demais aspectos da vida humana, quer seja numa visão macro ou a partir de um ponto de vista local (STOTZ, 1993; BLEIL, 1998; WEITZMAN, 2008).

No âmbito da ação extensionista que fundamentou este trabalho procurou-se desenvolver oficinas junto à população do entorno de uma clínica escola objetivando promover ações em educação e saúde na esfera nutricional, no período de março a julho de 2015. As oficinas foram desenhadas a partir de respostas obtidas através de um questionário aplicado previamente junto aos usuários da clínica. A sistematização do conjunto dos dados alcançados na fase inicial e nas ações posteriores do projeto possibilitou traçar algumas linhas gerais relativas a hábitos dietéticos e condutas de consumo que, aliadas à literatura especializada, podem auxiliar no delineamento de padrões de consumo alimentar num plano mais geral, comparativamente. 
Percepção da qualidade alimentar entre os usuários de uma clínica-escola no interior da Bahia, Brasil

\section{MATERIAIS E MÉTODOS}

Este estudo deriva de um projeto de extensão intitulado "Enfermagem e educação em saúde no contexto alimentar", realizado no âmbito de um curso de graduação em enfermagem de uma Faculdade privada no Sul da Bahia. As atividades aconteceram no espaço físico da Clínica Escola da União Metropolitana de Educação e Cultura (UNIMEItabuna) situada dentro do Campus I da Faculdade, que registrou em média duzentos atendimentos diários no período em estudo.

A clínica escola presta atendimento de atenção básica, especializado e de reabilitação com funcionamento diário das 7:00 às 22:00. A pesquisa foi realizada nos turnos manhã e noite. Os adultos que participaram do projeto são moradores dos bairros vizinhos à Faculdade, como também acompanhantes de pacientes que foram encaminhados por médicos de outros distritos do município de Itabuna para tratamento em especialidades médicas, fisioterápica e psicológicas.

As atividades desenvolvidas no projeto tiveram como público (a) famílias de usuários hipertensos e/ou diabéticos adultos, vítimas de acidente vascular encefálico que fazem tratamento de reabilitação fisioterápico na Clínica Escola, (b) mães, pais, cuidadores e cuidadoras de crianças com sequelas de paralisia cerebral. As atividades iniciaram em março e finalizaram em julho de 2015. Foram preenchidos 570 questionários junto aos usuários da clínica e, destes respondentes, 96 indivíduos - 64 mulheres e 32 homens - participaram de todas as oficinas subsequentes.

Os instrumentos usados para a coleta de dados foram dois: um questionário de múltipla escolha aplicado na sala de espera da clínica, enquanto o pai, a mãe ou o cuidador esperava o paciente fazer a terapia do dia e, na fase seguinte, o caderno de campo nas oficinas, com o objetivo de registrar a dinâmica daquele contexto. Os usuários foram abordados de forma aleatória e foram excluídos os menores de 21 anos. Após explicação sobre objetivos, fases do projeto e a assinatura do termo de consentimento livre e esclarecido, entregava-se o questionário para ser respondido.

No questionário havia pontos a respeito de variáveis socioeconômicas e demográficas (idade, local de moradia, número de habitantes na casa, escolaridade, emprego e renda); costume alimentar (alimentos que consome nas principais refeições diárias); e o conhecimento sobre rótulo de embalagens de alimentos industrializados. Após a conclusão 
do questionário entregava-se um flyer ao participante com as datas, local e horários das oficinas.

A sistematização das respostas apresentadas no questionário foi feitas no programa Excel através de planilhas, gráficos e tabelas buscando descrever o perfil do usuário participante do projeto e os principais alimentos consumidos pelas respectivas famílias. A partir das respostas se elegeu os temas das oficinas que constituiu a segunda etapa do projeto.

As oficinas educativas aconteceram em salas de aula localizadas na clínica, reservadas antecipadamente para feitura das atividades do projeto de extensão. Basicamente consistiu no registro sistemático da conduta dos participantes durante as oficinas. Foram construídas maquetes de embalagens, cartazes gigantes de rótulos, banners, realizados workshops culinários, troca de receitas e apresentação de documentários como material didático. Constituiu em nove oficinas com duração de 50 minutos, planejadas a partir de três temas: "Entendendo sobre o rótulo, ele é nosso amigo"; "O sal e o açúcar: nem vilão nem mocinho" e "Alimentos industrializados ou naturais: a escolba é sua".

\section{RESULTADOS E ANALÍSES}

Os resultados obtidos neste trabalho apresentam dados incisivos a respeito dos hábitos alimentares do grupo e da relação que ele estabelece com as informações da rotulagem nutricional dos produtos industrializados.

A respeito do perfil socioeconômico dos participantes do projeto de extensão (570 pessoas), $62 \%$ tinha renda familiar de até um salário mínimo e 10\% declarou perceber mais de dois salários mínimos mensais. Dos entrevistados 54\% dizia residir com mais de quatro pessoas, $31 \%$ indicava ter pelo menos uma companhia em casa e apenas 5\% morava só. Quanto à escolaridade, 41\% tinha ensino fundamental, 37\% possuíam ensino médio completo e $19 \%$, ensino superior.

A respeito dos dados de hábitos alimentares (quadro 1), 98\% dos inquiridos diziam utilizar caldos industrializados para preparar as refeições diárias e uma proporção semelhante dizia não fazer uso de frutas e verduras, com a justificativa do custo elevado destes alimentos. Quase 2/3 da população avaliada não fazia o desjejum matinal. E os que o faziam 
consumiam preferencialmente pão francês, biscoito e café. Os alimentos menos consumidos foram aipim, cará e inhame.

Quadro 1. Perfil alimentar dos participantes do projeto de extensão na Clínica Escola

\begin{tabular}{|c|c|c|c|c|}
\hline \multirow[b]{2}{*}{ Refeição } & \multicolumn{2}{|c|}{ Local da Refeição } & \multirow{2}{*}{\multicolumn{2}{|c|}{ Preferência alimentar }} \\
\hline & $\begin{array}{l}\text { Na própria } \\
\text { Residência }\end{array}$ & $\begin{array}{c}\text { Fora da } \\
\text { Residência }\end{array}$ & & \\
\hline \multirow{9}{*}{$\begin{array}{l}\text { Café da } \\
\text { Manhã }\end{array}$} & \multirow{9}{*}{$81 \%$} & \multirow{9}{*}{$19 \%$} & Pão & $95 \%$ \\
\hline & & & Biscoito & $95 \%$ \\
\hline & & & Café & $90 \%$ \\
\hline & & & Leite & $86 \%$ \\
\hline & & & Iogurte & $81 \%$ \\
\hline & & & Margarina & $83 \%$ \\
\hline & & & Queijo & $70 \%$ \\
\hline & & & Inhame, Cará, Aipim & $02 \%$ \\
\hline & & & Não faz esta refeição & $69 \%$ \\
\hline \multirow{7}{*}{ Almoço } & \multirow{7}{*}{$58 \%$} & \multirow{7}{*}{$42 \%$} & Feijão & $99 \%$ \\
\hline & & & Arroz & $99 \%$ \\
\hline & & & Carne vermelha & $99 \%$ \\
\hline & & & Carne branca & $96 \%$ \\
\hline & & & Macarrão & $89 \%$ \\
\hline & & & Salada & $80 \%$ \\
\hline & & & Não faz esta refeição & $10 \%$ \\
\hline \multirow{5}{*}{ Jantar } & \multirow{5}{*}{$89 \%$} & \multirow{5}{*}{$11 \%$} & Mesma refeição do almoço & $70 \%$ \\
\hline & & & $\begin{array}{l}\text { Mesma refeição do café da } \\
\text { manhã }\end{array}$ & $60 \%$ \\
\hline & & & Um lanhe rápido & $75 \%$ \\
\hline & & & Sopa/Caldo & $80 \%$ \\
\hline & & & Não faz esta refeição & $20 \%$ \\
\hline \multirow{4}{*}{ Lanches } & \multirow{4}{*}{$35 \%$} & \multirow{4}{*}{$65 \%$} & Refrigerante & $90 \%$ \\
\hline & & & Biscoito, salgadinho & $69 \%$ \\
\hline & & & Fruta/suco de Fruta & $10 \%$ \\
\hline & & & Não faz esta refeição & $87 \%$ \\
\hline
\end{tabular}

Fonte: dados da pesquisa

No almoço o feijão, o arroz e a carne vermelha eram alimentos quase unânimes. Para o jantar, verificou-se a tendência de repetir o cardápio do almoço ou do café da manhã. Nas 
oficinas foi recorrente depoimentos a respeito do desconhecimento do uso de ervas aromáticos como salsa e coentro, facilmente disponíveis em mercados e feiras livres locais.

O item salada foi assinalado como de consumo regular por $80 \%$ dos pesquisados, porém o seu conteúdo reduz-se a tomate, cebola e alface. As verduras eram consumidas principalmente junto à carne e costumeiramente cozidas, mormente batata inglesa, cenoura e abóbora. 90\% das pessoas diziam consumir refrigerantes nos lanches e 69\%, biscoito e/ ou salgadinhos. Nesta refeição, $10 \%$ indicavam consumir fruta ou suco de fruta e $87 \%$ dizia não ter costume em fazer esta refeição.

Outro ponto importante neste trabalho foi a verificação dos padrões de conduta a respeito da leitura e do entendimento dos rótulos dos produtos alimentares. A grande maioria - quase $80 \%$ dos participantes - disse nunca ter tido o interesse em ler os rótulos dos alimentos. Apenas 1\% lê e entende a descrição da rotulagem (quadro 2):

Quadro 2: Leitura e conhecimento dos rótulos de alimentos segundo os participantes do projeto de extensão na Clínica Escola

\begin{tabular}{|l|c|}
\hline Costuma ler e entende o que está escrito & $01 \%$ \\
\hline Lê ocasionalmente e nem sempre entende o que está escrito & $20 \%$ \\
\hline Nunca lê o que está escrito & $79 \%$ \\
\hline \hline Total & $100 \%$ \\
\hline \multicolumn{2}{|c|}{ Fonte: dados da pesquisa }
\end{tabular}

A partir dos resultados dos questionários, conforme planejamento prévio, foi desenhada e efetivada a primeira oficina, com o nome de "Entendendo sobre o rótulo, ele é nosso amigo". Foram elaborados banners com rótulos de diferentes alimentos e apresentado o documentário “Educação Nutricional: Rótulo dos Alimentos” (2012). O rótulo foi apontado pelos participantes como algo "difícil de entender", com "letras muito miúdas" e tendo "palavras estranhas". Ao serem solicitados a escolher um alimento a partir do que estava escrito no rótulo, expressavam: "não consigo" ou "não entendo". Observou-se, também, a que os participantes da oficina não conseguiam relacionar as informações contidas nos rótulos como um recurso para classificar o produto como algo "bom ou ruim" para a saúde.

Na semana seguinte foi realizada a segunda oficina, "O sal e o açúcar: nem vilão nem mocinho" onde foram apresentadas embalagens gigantes de alimentos e o documentário "De volta para o fogão" (2014). Buscou-se nesta oficina aprofundar a abordagem sobre o 
conhecimento dos rótulos, tratando de alguns dos elementos mais comuns nos rótulos: o sal $(\mathrm{NaCl})$ e o açúcar.

Os participantes se mostraram surpresos com as quantidades de sal e açúcar dos alimentos relacionados. Por exemplo, a baixa quantidade de cacau e a elevada quantidade de açúcar descritos nos rótulos dos achocolatados. Esta dinâmica consistiu em colocar em recipientes transparentes a mesma quantidade dos itens descritos nos rótulos. Neste caso, açúcar e cacau em pó. O mesmo foi feito para outros produtos como biscoitos, sucos e refrigerantes, especialmente em relação aos teores de sal e açúcar.

Neste momento foi comum interrogarem "para que o açúcar e o sal?”, "por que tal ou tal quantidade de sal ou de açúcar?”, “então o que eu bebo é suco de fruta e sal?”, referindo-se aí ao suco industrializado com extrato de soja. Após as dinâmicas os participantes das oficinas conseguiram identificar o sal e o açúcar em rótulos de embalagens e apontar alternativas à alimentação industrializada no debate após o documentário.

$\mathrm{Na}$ terceira oficina, intitulada "Alimentos industrializados ou naturais: a escolha é sua", buscou-se abordar a valorização dos saberes alimentares tradicionais. Tratou-se, também, do aproveitamento e descarte de alimentos, da viabilidade de construção de minihortas domésticas com base na permacultura e de receitas culinárias de baixo custo e de rápido preparo.

Neste momento do projeto, houve troca de receitas culinárias entre os participantes e dinâmicas onde foram selecionadas receitas para o café da manhã, lanche, almoço e jantar, com especial atenção para o lanche de crianças em fase escolar, a pedido de mães presentes. Também foi incentivada a recuperação de receitas tradicionais com ingredientes disponíveis na região.

Uma das hipóteses do projeto de extensão propunha que o indivíduo solteiro que vive só teria menos cuidado alimentar do que aquele que vive no âmbito de um núcleo familiar mais ampliado. Esta proposição não se verificou, uma vez que, de acordo com os questionários aplicados e as dinâmicas nas oficinas, não há uma relação entre a quantidade de membros da família e uma seletividade alimentar.

Outra linha que se seguiu foi a de que, corroborando a literatura especializada (LONGO-SILVA et al, 2015; MORAES, 2015; MUNIZ, 2015), há uma relação direta entre o perfil socioeconômico e a conduta a respeito do consumo de alimentos. Ao se considerar os 
Percepção da qualidade alimentar entre os usuários de uma clínica-escola no interior da Bahia, Brasil

dados obtidos nas atividades realizadas esta proposição se verificou integralmente: os indivíduos com menor renda e escolaridade tendem a (i) desconhecer os riscos de uma má alimentação à sua saúde e a (ii) não considerar as informações dos rótulos dos produtos alimentares, ignorando o que está ingerindo.

O plano de fundo do desenvolvimento de tais hábitos alimentares é, como ressaltam diversos autores, um processo mais amplo de mudanças socioculturais e econômicas que provoca a diluição dos vínculos culturais e afetivos com as referências baseadas na tradição de natureza familiar e/ou na coletividade local (CANESQUI e GARCIA, 2005; LEITE, 2007; FONSECA et al, 2009). Tem-se, portanto, o desenho da trajetória de uma desvinculação dos marcos culturais transmitidos por gerações e uma rápida incorporação de novos padrões alimentares - entre outros - fixados pelos mass media, pelo sistema produtivo industrial, por novos ritmos baseados no trabalho assalariado e por novos estilos de vida (TEIXEIRA et al, 2012; RUCKERT e GAIA, 2014; KLIEMANN et al, 2016).

No caso específico da região sul da Bahia, onde predominava de fins do século XIX até o começo da década de 1990 uma pujante lavoura cacaueira como lastro econômico, social e cultural, tem-se um acelerado processo migratório de trabalhadores rurais das fazendas devastadas pela praga vassoura de bruxa para os principais centros urbanos da região (Ilhéus, Itabuna e Porto Seguro) e alhures (FREITAS E PARAÍSO, 2001; FALCÓN, 1995). Este movimento demográfico vai provocar uma urbanização desordenada com uma rápida incorporação - simultaneamente ao esgarçamento dos laços tradicionais - de novos padrões comportamentais dentro de um retículo social geralmente insalubre, permeado pela carência material, sanitária e de informações básicas norteadoras de uma vida urbana sadia.

\section{CONSIDERAÇÕES FINAIS}

Os alimentos não se resumem a meros veículos de nutrientes. Eles trazem significações socioculturais, comportamentais e afetivas, podendo estabelecer importantes sentidos de pertencimento. Contudo, com o deslocamento histórico da centralidade da comensalidade no âmbito familiar, seguiu-se um novo estilo de vida com novos ritmos e novas prioridades e, com ele, novos problemas que repercutem no âmbito alimentar e nutricional (CANESQUI, 1988; GRAZZINELLI et al,, 2005; MOREIRA, 2010; SCHEK, BARBIER e HECK, 2015). 
Percepção da qualidade alimentar entre os usuários de uma clínica-escola no interior da Bahia, Brasil

No espaço da ação extensionista relatada, observou-se entre os participantes um acentuado desconhecimento do que se come e as virtuais consequências advindas do consumo de alimentos industrializados, segundo uma capacidade limitada de relacionar os agravos à saúde com a qualidade dos alimentos consumidos.

À luz da literatura especializada verifica-se, pois, a importância de desenvolver mais trabalhos de natureza inter e multidisciplinar envolvendo profissionais tanto da área de ciências sociais quanto da área de ciências da saúde no esforço de uma abordagem plural e holística no sentido de delinear padrões alimentares no contexto brasileiro para melhor auxiliar na elaboração de políticas públicas específicas.

Do mesmo modo, é preciso que profissionais de saúde e gestores que atuam no campo da saúde pública tenham um olhar mais acurado e sensível a respeito do tema nutricional e seus desdobramentos, tanto na prática clínica quanto no âmbito comunitário. E especialmente ações de educação e saúde que podem vir a promover redução de custos públicos com tratamentos e sobretudo promover qualidade de vida à população como um todo.

\section{REFERENCIAS}

ANJOS, L.A. Obesidade e Saúde Pública. Rio de Janeiro: Editora Fiocruz, 2006.

BLEIL, S.I. O Padrão Alimentar Ocidental: considerações sobre a mudança de hábitos no Brasil Revista Cadernos de Debate. Núcleo de Estudos e Pesquisas em Alimentação da UNICAMP, v. 6, p. 1-25.

BOOG, M.C.F. Dificuldades Encontradas por Médicos e Enfermeiros na Abordagem de Problemas Alimentares. Revista de Nutrição, v.12, n.3, p. 261-272, 1999.

BRASIL. Ministério da Saúde. Secretaria de Atenção à Saúde. Departamento de Ações Programáticas Estratégicas. Diretrizes de atenção à reabilitação da pessoa com acidente vascular cerebral. Brasília, 2013.

CANESQUi, A. M., Antropologia e alimentação. Revista de Saúde Pública, São Paulo, v. 22, n. 3, p. 207-216, 1988.

CANESQUI, A.M.; GARCIA R. W. D.(org.). Antropologia e nutrição: um diálogo possível. Rio de Janeiro: Editora FIOCRUZ, 2005.

COELHO, L.G., et al. Hábitos alimentares e risco de doenças cardiovasculares em escolares de Ouro Preto, Minas Gerais. Revista de Nutrição, v. 28, n.2, p.133-142, 2015.

De volta para o fogão. Rio de Janeiro: TEDX TALKS, 2014.16:13. Disponível em: https://www.youtube.com/watch?v=OWZ3dHWqocg. Acesso em: fevereiro de 2015. 
Percepção da qualidade alimentar entre os usuários de uma clínica-escola no interior da Bahia, Brasil

Educação Nutricional: Rótulo dos Alimentos. São Paulo. USP. 2012.6:28. Disponível em: https://www.youtube.com/watch?v=ww34UrMsJFw. Acesso em: março de 2015.

FALCÓN, G. Os Coronéis do cacau. Salvador: Edufba/Ianamá, 1995.

FONSECA, A.B. Modernidade alimentar e consumo de alimentos: contribuições sócioantropológicas para a pesquisa em nutrição. Revista Ciência \& Saúde Coletiva, v.16, n.9, p.38533862, 2011.

FREITAS, A.; PARAÍSO, M. H. Caminhos ao Encontro do Mundo: a capitania, os frutos de ouro e a princesa do sul - Ilhéus 1534-1940. Ilhéus: Editus, 2001.

GRAZZINELLI, M.F., et al. Educação em Saúde: conhecimentos, representações sociais e experiência da doença. Caderno de Saúde Pública, v.21, n.1, p. 200-206. 2005.

KAC, G; et al. (org). Epidemiologia nutricional. Rio de Janeiro: Editora Fiocruz/Atheneu, 2007.

KLIEMANN, N. et al. Porção na rotulagem nutricional de alimentos processados comercializados no Brasil: relação com o valor energético. Revista de Nutrição, v.29, n.5, p.741-750, 2016.

LAPLANTINE, F. Antropologia da doença. 4a edição. São Paulo: WMF Martins Fontes, 2010.

LIMA, L.A. Hábitos alimentares de hipertensos e diabéticos atendidos em um serviço de Atenção Primária à Saúde do Sul do Brasil. Revista de Nutrição,v. 28. n. 2, p.197-206, 2015

LONGO-SILVA, G., et al. Alimentos ultra-processados: consumo entre crianças em creches públicas e análise da composição nutricional segundo a ferramenta "Traffic Light Labelling". Revista de Nutrição, v. 28, n.5, p.543-553, 2015.

MENDES, E.V. A APS no Brasil. In: Mendes EV, org. O cuidado das condições crônicas na atenção primária à saúde: o imperativo da consolidação da estratégia da saúde da família. Brasilia, DF: Organização Pan-Americana da Saúde. p. 71-99, 2012.

MORAES, B.V. et al., Desconhecimento da hipertensão arterial e seus determinantes em quilombolas do sudoeste da Bahia, Brasil. Revista Ciência \& Saúde Coletiva, v. 20, n.3, p.797-807, 2015.

MOREIRA, S.A. Alimentação e comensalidade: aspectos históricos e antropológicos. Revista Ciência e Cultura. v. 62, n.4, p. 23-26, 2010.

MUNIZ, V.M., et al. Consumo alimentar de famílias de trabalhadores de cana-de-açúcar. Revista de Nutrição, v.28, n.2, p.175-184, 2015.

NAJAS, M.S., et al. Padrão alimentar de idosos de diferentes estratos socioeconômicos residentes em localidade urbana da região sudeste, Brasil. Revista de Saúde Pública, v. 28, n.3, p.187- 191,1994.

PINHEIRO, A., et al. O que é uma alimentação saudável: considerações sobre o conceito, princípios e características: uma abordagem ampliada. Ministério da Saúde, Brasília, 2005.

POSSA, G., et al. Consumo de lácteos e derivados no Brasil está associado com fatores socioeconômicos e demográficos: resultados do Inquérito Nacional de Alimentação 2008-2009. Revista de Nutrição, v.30, n.1, p.79-90, 2017.

ROMANELLI, G.O. Significado da alimentação na família: uma visão antropológica. Revista de Medicina, v.39, n.3, p. 333-339, 2006. 
RUCKERT, B.; GAIA, M.C.M. Educação Popular, saúde e segurança alimentar em áreas do movimento dos trabalhadores rurais sem terra. Tempus, Acta de Saúde Coletiva, Brasília, p. 57171, 2014.

SCHEK, G., et al. Processo saúde/doença e Cuidado em famílias descendentes de pomeranos: contribuições para a enfermagem. Revista de Enfermagem, v. 11, n. 11, p.54-62, 2015.

RÜCKERT, B.; GAIA, M.C.M. Educação popular, saúde e segurança alimentar em áreas do Movimento dos Trabalhadores Rurais Sem Terra. Actas de Saúde Coletiva, v.8, n.2, p. 157-171, 2014.

SOARES, L.M. Transformação e Persistência: antropologia da alimentação e nutrição em uma sociedade indígena amazônica. Rio de Janeiro: Editora Fiocruz, 2007.

STOTZ, E.M. Enfoques sobre a educação e saúde. In: VALLA, V. \& STOTZ, E. N. (orgs) Participação popular, Educação e Saúde: teoria e prática. Rio de Janeiro: Relume-Dumará, p.11-22. 1993.

TEIXEIRA, M.G., et al. Medidas de proteção na promoção de alimentação saudável: uma revisão de literatura. Revista Brasileira de Pesquisa em Saúde, v.14, n.4, p. 90-96, 2012.

WEITZMAN, R. Educação Popular em Educação Alimentar e Nutricional: uma metodologia de formação com enfoque no gênero. Rede de intercâmbio de Tecnologias Alternativas. Belo Horizonte: 2008. 